\title{
Hybrid PMM-MoM Method for the Analysis of Finite Periodic Structures
}

\author{
Shan Zhao,Naiqian Zhang,Dong Li \\ Information Engineering College \\ Communication University of China \\ Beijing, China \\ zhaos@cuc.edu.cn,ddznq@cuc.edu.cn, lidong@cuc.edu.cn
}

\author{
Jianxun $\mathrm{Su}$ \\ Department of Microwave Technology \\ East China Research Institute of Electronic Engineering \\ Hefei, China \\ sujianxun_jlgx@163.com
}

\begin{abstract}
In this paper, a hybrid method (hybrid PMM-MoM method) is presented for the effective and accurate analysis of finite periodic structures. This method divides a finite periodic structure into two parts. The inner part of an approximate infinite periodic structure is analyzed by periodic method of moment (PMM); the outer part is then analyzed by method of moments (MoM). For the finite periodic structures, the accuracy of the new method is much better than that of the pure PMM, and is almost the same as that of pure MoM. Because pure PMM uses the periodic boundary conditions, it takes much less memory resources and computation time. For hybrid PMM-MoM method, because the inner part is calculated by PMM, the calculation work concentrates on the outer part. Consequently, compared with the exact MoM, the new method saves much more memory resources and computation time, which provides a drastic reduction of unknowns.
\end{abstract}

Keywords-hybrid method; PMM-MoM; infinite Periodic Structures; RCS

\section{Introduction}

A hybrid PMM-MoM (periodic MoM and exact MoM) method is proposed for the analysis of arbitrary finite periodic structures. Surface waves are unique for finite periodic structures, which will not appear in the infinite one, and the surface waves and Floquet currents in this case will interfere with each other, resulting in strong variations of the current amplitudes [1]. Therefore, if modeling finite periodic structures by PMM [2], it will cause significant errors or even lead to wrong results sometimes. The exact full-wave model is employed in the analysis of finite periodic structures, including both planar and curved structures. However, the strict model takes up a great deal of memory, and computing time is also unacceptable, especially for large finite periodic structures. Therefore, a new method to save memory and to obtain sufficient accuracy is presented for the analysis of finite periodic structures. The new method divides the finite periodic structures into two parts. The inner part of an approximate infinite periodic structure is analyzed by pure PMM, and the outer part polluted by edge effect is analyzed by the exact full-wave model, i.e, MoM. The new method can obtain sufficient accuracy and save significant memory and computation time.

\section{Formulation}

\section{A. Structural Analysis}

Consider a finite periodic structure under the illumination of the plane wave with polarization direction along the $\mathrm{x}$-axis showed in Fig. 1. The structure can be divided into two parts, the inner part and the outer part. Suppose that the inner array elements are unaffected by the edge effects, then the periodic boundary conditions hold true for the inner part so that PMM can be used. When the division is made properly, this will introduces relatively very small errors. On the other hand, for the outer part, the edge effects can not be ignored, and it must be analyzed by the exact full-wave methods such as MoM, etc. Due to the high efficiency of PMM, the hybrid PMM-MoM method can save much more memory and computation time compared with exact MoM.

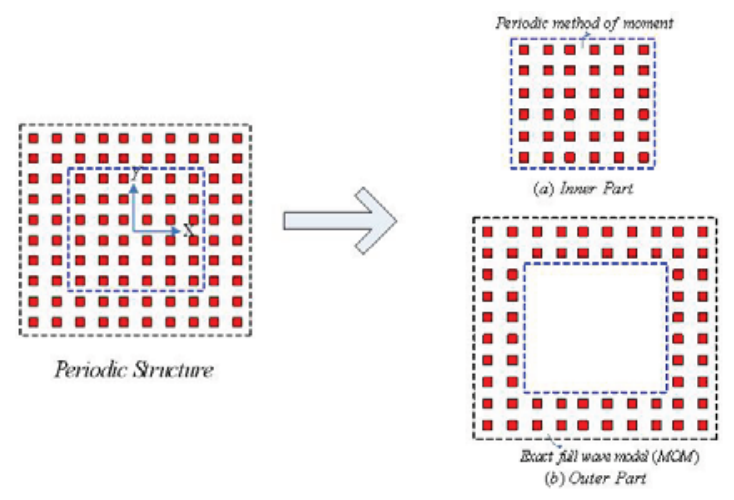

Figure 1. A finite periodic structure divided into two parts

\section{B. Solution Scheme}

Analysis steps of hybrid PMM-MoM method for the finite periodic structure shown in Fig.1 can be described as follows.

1) Solution of Infinite-periodic Integral Equation for Inner Part

Since the inner part is little influenced by the edge effects, it can be treated as an infinite periodic structure. Periodic boundary conditions allow the reduction of computational complexity of the inner part almost to a single elementary cell. Regardless of the element shape,

This work is Sponsor by International Cooperation Projects (AM0552) 
vector spectral-domain method [3] is used to solve the infinite periodic integral equation. For a free-standing 2-D planar infinite periodic structure illuminated by a plane wave, the incident filed and the induced currents on the conducting surfaces are related by the equation $[3,4]$ :

$$
\begin{gathered}
-\vec{E}^{i n c}=\frac{1}{j \omega \varepsilon} \sum_{m=-\infty}^{\infty} \sum_{n=-\infty}^{\infty}\left[\begin{array}{cc}
k_{0}^{2}-\alpha_{m n}^{2} & -\alpha_{m n} \beta_{m n} \\
-\alpha_{m n} \beta_{m n} & k_{0}^{2}-\beta_{m n}^{2}
\end{array}\right] \tilde{\overline{\vec{G}}}\left(\alpha_{m n}, \beta_{m n}\right) \\
\cdot \tilde{\vec{J}}\left(\alpha_{m n}, \beta_{m n}\right) e^{j \alpha_{m n} x} e^{j \beta_{m n} y}
\end{gathered}
$$

where $\vec{E}^{\text {inc }}$ represents the incident filed, $\tilde{\tilde{J}}\left(\alpha_{m n}, \beta_{m n}\right)$ and $\tilde{\overline{\vec{G}}}\left(\alpha_{m n}, \beta_{m n}\right)$ represent the induced current and the green's function in the spectral domain with $(\mathrm{m}, \mathrm{n})$ Floquet harmonic, respectively. In addition, $\alpha_{m n}=\frac{2 m \pi}{T_{x}}-k_{x}^{i n c}, \beta_{m n}=\frac{2 n \pi}{T_{y}}-k_{y}^{i n c}$, where $T_{x}$ and $T_{y}$ are the periods in the $x, y$ directions, respectively, $k_{x}^{i n c}$ and $k_{y}^{i n c}$ are given by $k_{x}^{i n c}=k_{0} \sin \theta_{i} \cos \varphi_{i}$ and $k_{y}^{i n c}=k_{0} \sin \theta_{i} \sin \varphi_{i},\left(\theta_{i}, \varphi_{i}\right)$ are the incident angles of the plane wave, $k_{0}$ is the free-space wavenumber, and

$$
\tilde{\overline{\vec{G}}}\left(\alpha_{m n}, \beta_{m n}\right)=\frac{-j}{2 \sqrt{k_{0}^{2}-\alpha_{m n}^{2}-\beta_{m n}^{2}}} \overrightarrow{\bar{I}}
$$

where $\overrightarrow{\vec{I}}$ is identity tensor.

Compute the induced current of inner part by PMM, under the illumination of the plane wave.In PMM, proper choice of basis functions is very important $[5,6]$, in the following analysis, the induced surface currents are expressed in terms of RWG basis functions.

2) Solution of (Electric) Field Integral Equation for Outer Part

Compute the induced current (JCurrent1) of outer part by traditional MoM. In this case, there are two excitation sources show in Fig. 2, i.e., the plane wave and JCurrent 2 .

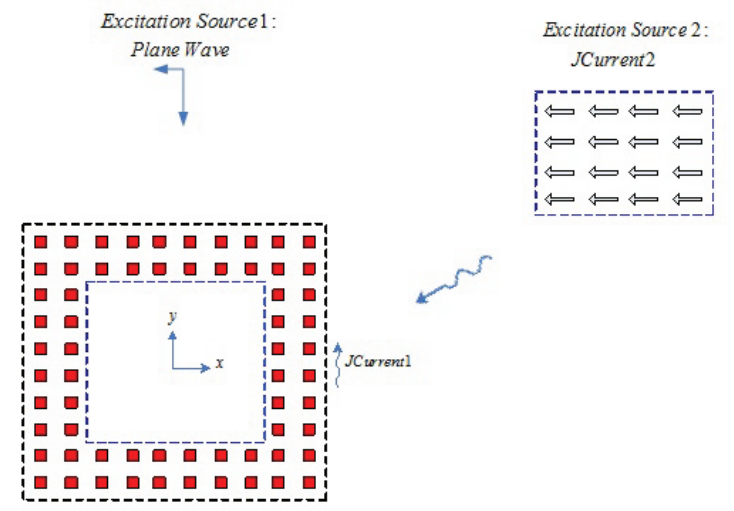

Figure 2. Outer part is excited by plane wave and JCurrent 2 at the same time
For the outer part, the perfectly conducting boundary condition can be used to derive the following electric field integral equation (EFIE) [7]:

$\hat{t} \cdot j k \int_{S} d^{\prime \prime} \eta\left[J\left(\vec{r}^{\prime}\right)+\frac{1}{k^{2}} \nabla^{\prime} \cdot J\left(\vec{r}^{\prime}\right) \nabla\right] G\left(\vec{r}, \vec{r}^{\prime}\right)=-t \cdot \eta^{-1} \vec{E}^{i n c}(\vec{r})($ 3)

where, the time factor $e^{-j \omega t}$ is employed. In (3), the scattered filed is expressed in terms of the induced (unknown) surface current $\vec{J}, \vec{r}$ and $\vec{r}^{\prime}$ represent the observation point and the source point on the surface, respectively, $\hat{t}$ is the unit tangential vector of the surface at the observation point, $\vec{E}^{i n c}$ is the incident electric field, $\eta$ is the wave impedance of the medium space, $k=\omega \sqrt{\varepsilon \mu}=2 \pi / \lambda$ is the wavenumber, and $G\left(\vec{r}, \vec{r}^{\prime}\right)=\frac{e^{-j k R}}{4 \pi R} \quad\left(\mathrm{R} \overrightarrow{\boldsymbol{F}}-\vec{r}^{\prime} \mid\right)$ denotes the homogeneousspace Green's function.

3) Solution of Truncate Edge Effects

By the physical optics approximation, the current density on the illuminated side of the scatter has the value $\vec{J}_{S}=2\left(\vec{n} \times \vec{H}^{i n c}\right)$. For the above-mentioned scattering array shown in Fig.1, the induced current flows mainly along $\mathrm{x}$ axis. As is well known, the wire current has much stronger radiation in the plane perpendicular to its flowing direction than that along the flowing direction, so that the coupling effects among the array elements in y-direction (vertical direction) are stronger than those in x-direction (horizontal direction). Therefore, the number of columns polluted by the edge effects on the left and right edges of the array is less than the number of rows polluted by the edge effects on the upper and lower edges of the array. Fig. 3 shows current density distribution of $21 \times 21$ patch array. The area surrounded by red line is almost not affected by the edge effects. Thus, the inner part with the characteristics of infinite periodic structure can be analyzed by PMM. However, the area outside the red line is polluted seriously by edge effect. Outer part must be analyzed by exact fullwave model.

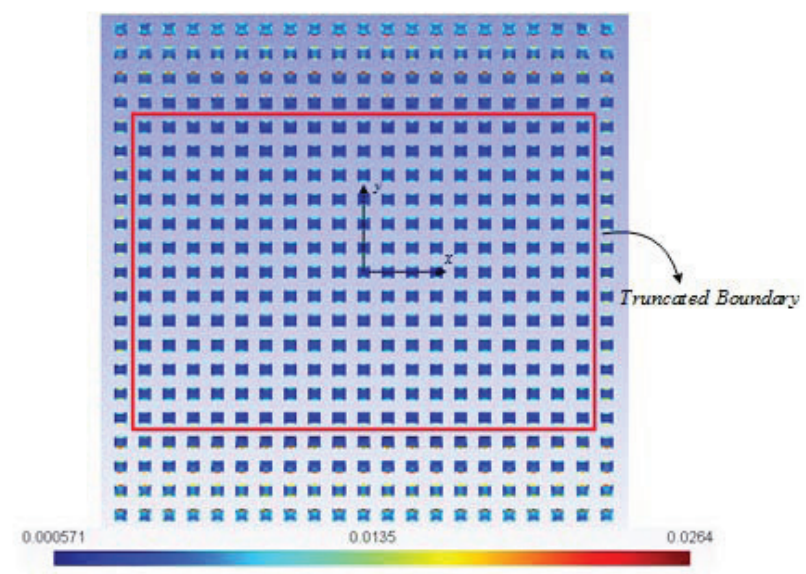


Figure 3. Current density distribution of $21 \times 21$ patch array

Through numerical tests, it is found that the size of the area affected by edge effects is mainly determined by the inter-element spacing, size and shape of the element, and is almost not related to the size of the array. The truncated row number can be fixed approximately as twice as the truncated column number.

The size of truncated area can be evaluated roughly in advance. Generally, the truncated column and row numbers can be set to 2 and 4 . If the scale of the scattering array is more than $31 \times 31$, the truncated column and row numbers can also be set to 3 and 5. The back-scattered RCS is calculated by the hybrid PMM-MoM method and noted as $R C S_{\text {before }}$. Then, the truncated column and row numbers are increased by 1 and 2, respectively. The back-scattered RCS is calculated again by the hybrid PMM-MoM method and noted as $R C S_{\text {after }}$. If $\left|R C S_{\text {before }}-R C S_{\text {after }}\right|<\Delta$, the results can be considered as convergent. A small enough $\Delta$ value should be chosen according to the accuracy requirement. If $\left|R C S_{\text {before }}-R C S_{\text {affer }}\right|>\Delta$, the truncated column and row number should be increased in the further computation until the satisfactory results are obtained.

\section{Numerical Results}

In this section, the scattering of finite periodic array is analyzed by the hybrid PMM-MoM method. To evaluate this new method, consider two arrays: a small planar array of dipoles and a large planar array of patches.

\section{A. $21 \times 21$ dipole array}

The planar dipoles are used as the array elements. First, use ANSYS to mesh the array element at the lower left corner into Delaunay triangles shown in Fig.4. Then, the triangle mesh info of this array element is cloned by a short program to obtain the triangle mesh info of the whole periodic structure. In this way, all the array elements have the same triangle mesh.

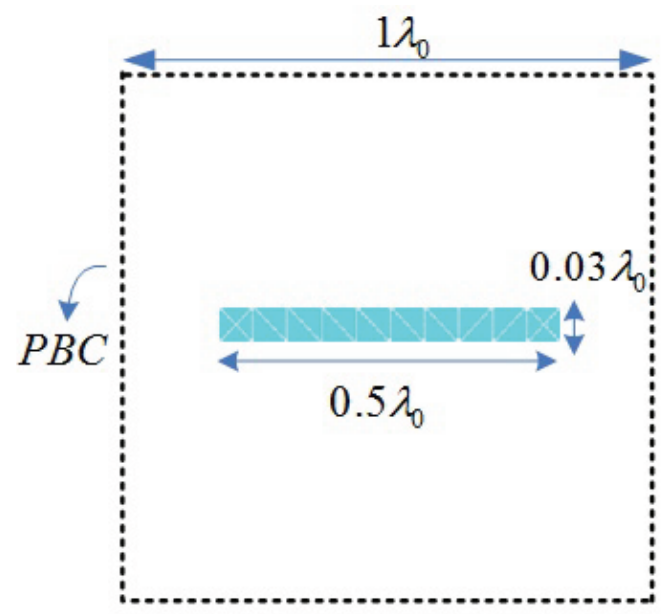

Figure 4. Discretized unite cell with period $p_{x}=p_{y}=1 \lambda_{0}$
In the first calculation, the truncated column and row number are set to 2 and 4. The back-scattered RCS calculated by the hybrid PMM-MoM method is $40.0138 \mathrm{~dB}$. In the next calculation, the truncated column and row number are increased by 1 and 2 to 3 and 6, respectively. The calculation result of the hybrid PMM-MoM method is 40.9721dB. Since $\left|\mathrm{RCS}_{\text {before }}-\mathrm{RCS}_{\text {after }}\right|=0.9583<1 d B$, the numerical results are considered as convergent. The numerical results are shown in Fig.5.

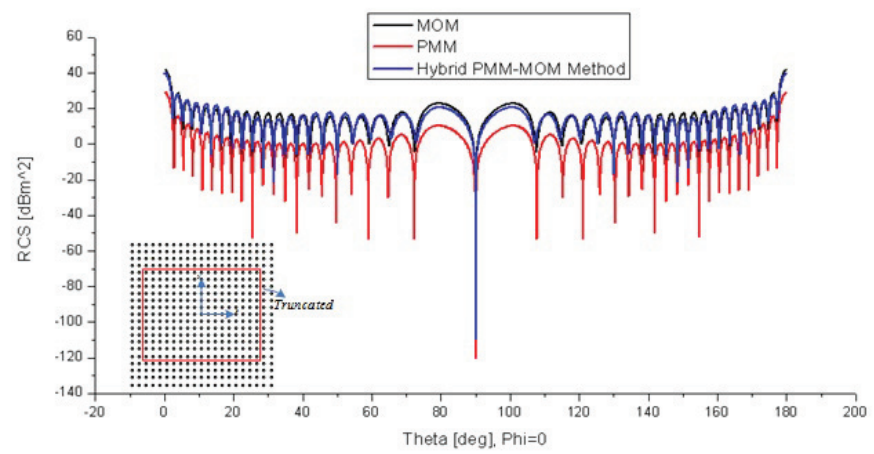

Figure 5. RCS of a $21 \times 21$ dipole array under the illumination of a plane wave(Two columns and four rows are truncated on the array boundary)

From Fig.5, where two columns and four rows are truncated for the outer part, it can be seen that, PMM results have $20 \sim 60 \mathrm{~dB}$ difference from those of the exact MoM while the results obtained by the hybrid PMM-MoM method show very good agreement. Comparison of the memory and computing time for MoM and hybrid PMMMoM method is shown in Table I.

TABLE I. COMPARISON OF MEMORY AND COMPUTING TIME FOR MOM AND HYBRID PMM-MOM METHOD

\begin{tabular}{|l|c|c|}
\hline & MOM & Hybrid PMM-MOM Method \\
\hline Memory/Mb & 935 & 234 \\
\hline CPU/Second & 375 & 143 \\
\hline
\end{tabular}

In Table I, the whole dipole array has 441 dipole elements and is discretized into 10584 Delaunay triangles. The RWG basis function number is 11025 . Two columns and four rows are truncated for the outer part. The outer part and inner part have 220 and 221 dipole elements, respectively. It should be noticed that the hybrid PMMMoM method implies the solution of a $220 \times 220$ linear system, while the exact MoM approach solves a $441 \times 441$ linear system.

\section{B. $51 \times 51$ patch array}

Unite cell is discretized into Delaunay triangles show in Fig.6, consider the planar array composed of the square patches with side length $0.5 \lambda_{0}$. The period is $p_{x}=p_{y}=1 \lambda_{0}$. Because the electric size of the array is quite large, the hybrid PMM-MoM method and the exact 
MoM approach are both accelerated by MLFMA (Multilevel Fast Multipole Algorithm) [8].

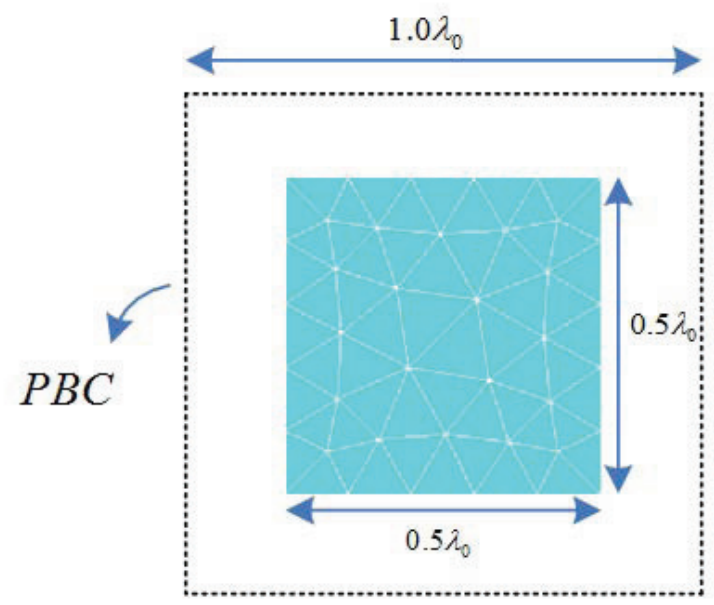

Figure 6. unite cell is discretized into Delaunay triangles

Similarly, in the first calculation, the truncated column and row number are set to 2 and 4 . The back-scattered RCS calculated by the hybrid PMM-MoM method is $64.01141 \mathrm{~dB}$. In the next calculation, then, the truncated column and row number are increased by 1 and 2 to 3 and 6 , respectively. The result of the hybrid PMM-MoM method is now $64.8018 \mathrm{~dB}$. Since $\left|\mathrm{RCS}_{\text {before }}-\mathrm{RCS}_{\text {after }}\right|=0.79039<1 d B$, the results can be considered as convergent. The numerical results are shown in Fig.7.

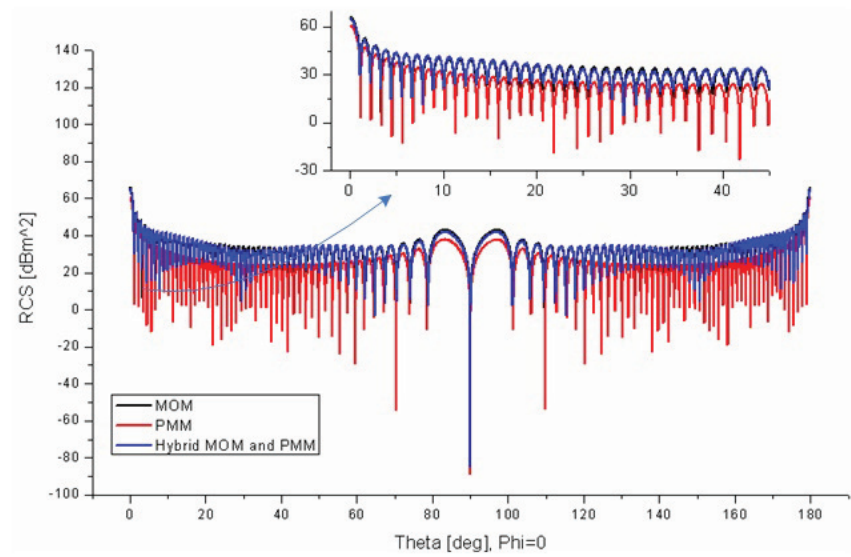

Figure 7. RCS of a $51 \times 51$ patch array under the illumination of a plane wave(Two columns and four rows are truncated on the array boundary)

In Fig.7, two columns and four rows are truncated for the outer part, in which 580 elements are included. Excellent agreement between the results of the hybrid PMM-MoM method and the exact MoM is observed. On the other hand, PMM results have $10 \sim 60 \mathrm{~dB}$ difference from those of the exact MoM. We emphasize again that the hybrid PMM-MoM method implies the solution of a $580 \times 580$ linear system, while the exact MoM approach solves a $2601 \times 2601$ linear system.

\section{Conclusion}

Conventionally, a finite periodic structure such as an FSS or antenna array [9] is considered as an infinite one and analyzed by PMM. In this paper, a hybrid PMM-MoM method is proposed to calculate the RCS of two finite planar arrays under the illumination of a plane wave. Significant errors are shown in this case when using the pure PMM. The results obtained by the hybrid PMM-MoM method agree very well with those of the exact MoM. Meanwhile, compared with the exact MoM, the new hybrid method drastically reduces the scale of solving linear system. So, hybrid method shows much higher efficiency and saves much more memory resources and calculation time.

\section{References}

[1] Munk B A. Finite Array Antennas and FSS [M], New York: Wiley, 2003.

[2] R. Mittra, C. H. Chan. T. Cwik, "Techniques for analyzing frequency selective surface- a review," Proceeding of the IEEE, vol. 76, no. 12, 1988, pp. 1593-1615.

[3] A. Qing, "VECTOR SPECTRAL-DOMAIN METHOD FOR THE ANALYSIS OF FREQUENCY SELECTIVE SURFACES", Progress In Electromagnetics Research, PIER 65, 201-232, 2006

[4] T. K. Wu, "Frequency selective surface and grid array," John Wiley \& Sons, Inc Wiley, 1995.

[5] Kim Mcinturff and Peter S. Simon, "The Fourier transform of linearly varying functions with polygonal support," IEEE Trans. Antennas and Propagation, vol. 39, no.9, 1991, pp.144 -1443.

[6] Chen, H., L. Ran, J. Huangfu, X. M. Zhang, K. Cheng, T. M. Grzegorczyk, and J. A. Kong, "Magnetic properties of S-shaped split-ring resonators," Progress In Electromagnetics Research, PIER 51, 231-247, 2005.

[7] S. M. Rao, D. R. Wilton, and A. W. Glisson, "Electromagnetic scattering by surface of arbitrary shape,"IEEE Trans. Antennas and Propagation, vol. 30 no.3, 1982, pp.409-411.

[8] J. M. Taboada, M. G. Araujo, J. M. Bertolo, L. Landesa, F. Obelleiro, and J. L. Rodriguez, "MLFMAFFT Parallel Algorithm for the Solution of LargeScale Problems in Electromagnetics(INVITED PAPER)", Progress In Electromagnetics Research, PIER 105, 15-30, 2010.

[9] Tasinkevych. Y, "EM SCATTERING BY THE PARALLEL PLẢTE WAVEGUIDE ARRAY WITH THICK WALLS FOR OBLIQUE INCIDENCE", Journal of Electromagnetic Waves \& Applications, Sep2009, Vol. 23 Issue 11/12, pp.1611-1621. 


\title{
Description of the derived categories of tubular algebras in terms of dimension vectors
}

\author{
Hongbo Lv, Zhongmei Wang \\ School of Mathematical Science, University of Jinan, Jinan 250022, P.R.China \\ School of Control Science and Engineering, Shandong University, Jinan 250061, P.R.China \\ Email: lvhongbo356@163.com; wangzhongmei211@sohu.com
}

\begin{abstract}
In this paper, we give a description of the derived category of a tubular algebra by calculating the dimension vectors of the objects in it.
\end{abstract}

Keywords:derived categories; tubular algebra; dimension vectors

\section{Introduction}

Let $\Lambda$ be a basic connected algebra over an algebraically closed field $k$. We denote by $\bmod \Lambda$ the category of all finitely generated right $\Lambda$-modules and by ind $\Lambda$ a full subcategory of $\bmod \Lambda$ containing exactly one representative of each isomorphism class of indecomposable $\boldsymbol{\Lambda}$-modules. For a $\boldsymbol{\Lambda}$-module $\boldsymbol{M}$, we denote the dimension vector by $\operatorname{dim} \boldsymbol{M}$. The bounded derived category of mod $\Lambda$ is denoted by $\boldsymbol{D}^{t}(\boldsymbol{\Lambda})$. We denote the Grothendieck group of $\Lambda$ by $\boldsymbol{K}_{0}(\Lambda)$, AuslanderReiten translation by $\tau$, the Cartan matrix by $C_{\Lambda}$. Let $\hat{\Lambda}$ be the repetitive algebra of $\Lambda$, mod $\hat{\Lambda}$ the stable module category. When the global dimension of $\Lambda$ is finite, $C_{\Lambda}$ is invertible by [1], and $\boldsymbol{D}^{b}(\boldsymbol{\Lambda})$ is equivalent to $\bmod \hat{\boldsymbol{\Lambda}}$ as triangulated categories by [2].

By [1], a tubular extension $A$ of a tame-concealed algebra

of extension type $\mathrm{T}=(2,2,2,2),(3,3,3),(4,4,2)$ or $(6,3$, 2)

is called a tubular algebra. For example, the canonical tubular algebras of $\mathrm{T}(2,2,2,2)$ is determined by the following quiver with relations.

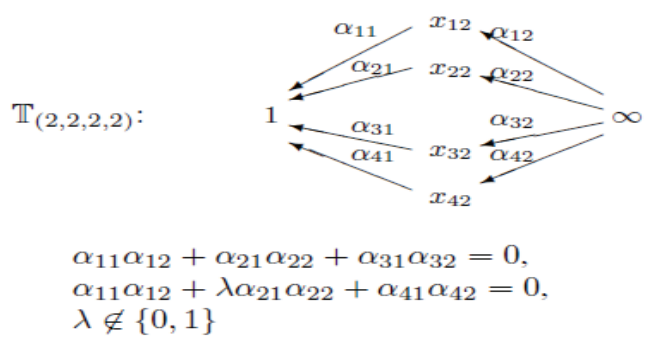

By [1], global dimension of a tubular algebra $A$ is 2 , then $\boldsymbol{D}^{b}(A)$ is equivalent to mod $\hat{A}$. And tubular algebras of the same extension type are tilt-cotilt equivalent, see [3]. Then we only consider the derived categories of canonical tubular algebras, whose structures are given in [4].

$$
D^{b}(A)=\underset{r \in Q}{\vee} T_{r}
$$

where (1) for any $r \in Q, T_{r}$ is the standard stable $\mathrm{P}_{1}(k)$ tubular family of type $\mathrm{T}$;

(2) for any $r \in Q, T_{r}$ is separating $\underset{s<r}{\vee} T_{s}$ from $\underset{r<u}{\vee} T_{u}$.

Based on the results above, we give a description of the derived category of a canonical tubular algebra by calculating the dimension vectors of the objects in it.

\section{Description of The Derived Categories of Tubular Algebras In Terms Of Dimension Vectors}

In this section, let $A$ be a canonical tubular algebra of type $\mathrm{T}$.

Definition 1.1. ([1]) Let $n$ be the rank of Grothendieck group $K_{0}(A), C_{A}$ the Cartan matrix of $A$. Then

(1) The Coxeter matrix $\Phi_{A}$ is defined by $-C_{A}{ }^{-T} C_{A}$;

(2) The quadratic form $\chi_{A}$ in $\mathbb{Z}_{n}$ is defined by

$$
\chi_{A}(\alpha)=\frac{1}{2} \alpha\left(-C_{A}^{-T}+C_{A}^{-1}\right) \alpha^{T}
$$

for any $\alpha$ in $\mathbb{Z}_{n}$.

(3) Let $h_{0}, h_{\infty}$ be the positive generators of $\operatorname{rad} \chi_{A}$. For an $A$-module $M$, define

where

$$
\operatorname{index}(M)=-\frac{l_{0}(\underline{\operatorname{dim}} M)}{l_{\infty}(\underline{\operatorname{dim}} M)}
$$

$$
\begin{array}{r}
l_{0}\left(\underline{\operatorname{dim} M)}=h_{0} C_{A}^{-T}\left(\underline{\operatorname{dim} M)^{T},}\right.\right. \\
l_{\infty}\left(\underline{\operatorname{dim} M)=h_{\infty} C_{A}^{-T}(\underline{\operatorname{dim}} M)^{T} .}\right. \text { particular, } \\
\text { In }{ }_{\alpha \in \operatorname{rad}} \chi_{A}, \alpha=r_{0} h_{0}+r_{\infty} h_{\infty}, \\
r_{0}, r_{\infty} \in \mathbb{Z} . \text { Then, index }(\alpha)=\frac{r_{\infty}}{r_{0}} .
\end{array}
$$$$
\text { In particular, for any }
$$ 
(4) $\alpha \in \operatorname{rad} \chi_{A}$ is called a real (respectively, imaginary) root,

if $\chi_{A}(\alpha)=\frac{1}{2} \alpha\left(-C_{A}{ }^{-T}+C_{A}{ }^{-1}\right) \alpha^{T}=1$ (respectively, $=0$ ).

It is well known that there exists a "minimal " imaginary root $\delta$ such that $\operatorname{rad} \chi_{A}=\mathbb{Z} \delta$.

Now we recall some results in [4]. Let $\Lambda$ be a finite dimensional $k$-algebra and $\widehat{\Lambda}$ the repetitive algebra. Denote

by $P(\widehat{\Lambda})$ the subgroup of $K_{0}(\widehat{\Lambda})$ generated by the dimension vectors of indecomposable projective $\widehat{\Lambda}-$ modules.

Lemma 1.2. $K_{0}(\widehat{\Lambda})=K_{0}(\Lambda)+P(\widehat{\Lambda})$.

Definition 1.3. Let $\pi_{\Lambda}: K_{0}(\widehat{\Lambda}) \rightarrow K_{0}(\Lambda)$ be the projective morphism. Define $\underline{\operatorname{dim}}^{\Lambda}: \bmod \widehat{\Lambda} \rightarrow K_{0}(\Lambda)$ where for any $\widehat{\Lambda}$-module $X, \underline{\operatorname{dim}}^{\Lambda} X=\pi_{\Lambda}(\underline{\operatorname{dim}} X)$.

Lemma 1.4. Let $\Phi_{\Lambda}$ be the Coxeter matrix of $\Lambda, \hat{\tau}$ the Auslander-Reiten translation of $\widehat{\Lambda}$. Then

$$
\underline{\operatorname{dim}}^{\Lambda} \hat{\tau} X=(\underline{\operatorname{dim}} X) \Phi_{\Lambda} .
$$

Note that if $\Lambda$ has finite global dimension, we have a triangulated equivalence: $\eta: D^{b}(\Lambda) \rightarrow \underline{\bmod \widehat{\Lambda}}$. For an object $X^{\cdot} \in D^{b}(\Lambda)$, define $\underline{\operatorname{dim}} X^{\cdot}=\sum_{i}(-1)^{i} \underline{\operatorname{dim}} X^{i}$.

Then we have

Lemma 1.5. $\underline{\operatorname{dim}} X^{\cdot}=\underline{\operatorname{dim}}^{\Lambda} \eta\left(X^{\bullet}\right)$. [5]

By representation theory of Auslander-Reiten quivers in and the results above, we have a method to describing the derived category of a canonical tubular algebra in terms of dimension vectors.

Theorem 1.6. Let $A$ be a canonical tubular algebra of type

$\mathrm{T}$, the rank of $K_{0}(A)$ be $n$. Then

(1) Let $\hat{\delta}$ be the minimal imaginary root in $K_{0}(\widehat{A})$ corresponding the $\mathrm{P}_{1}(k)$ - tubular family $T_{r}$, and let $\delta=\underline{\operatorname{dim}}^{A}(\hat{\delta})$. Then $\delta$ is determined by $\chi_{A}(\delta)=0$.

(2) Let $X$ be an object in the bottom of a tube of rank $r$ in $T_{r}$. Then $\underline{\operatorname{dim}}^{A} X$ is determined by the following:

(*) $\left\{\chi_{A}\left(\underline{\operatorname{dim}}^{A} X\right)=\frac{1}{2} \underline{\operatorname{dim}}^{A} X\left(-C_{A}{ }^{-T}+C_{A}{ }^{-1}\right)\left(\underline{\operatorname{dim}}^{A} X\right)^{T}=1\right.$ $\underline{\operatorname{dim}}^{A} X+\left(\underline{\operatorname{dim}}^{A} X\right) \Phi_{A}+\cdots+\left(\underline{\operatorname{dim}}^{A} X\right) \Phi_{A}{ }^{r-1}=\delta$.
Proof. (1) By [4], $\delta=r_{0} h_{0}+r_{\infty} h_{\infty} \in \operatorname{rad} \chi_{A}$, and thus $\chi_{A}(\delta)=0 . \quad$ Since $\quad \operatorname{index}(\delta)=\frac{r_{\infty}}{r_{0}} \in \mathbb{Q}, \quad$ where $r_{0}, r_{\infty} \in \mathbb{Z}$, and $\left(r_{0}, r_{\infty}\right)=1$, it suffices to calculating $r_{0}$ and $r_{\infty}$.

(2) Directly from Lemma 1.4 and 1.5 .

Example 1.7. Now let $A$ be a canonical tubular algebra of type $\mathrm{T}(2,2,2,2)$. The Cartan matrix and Coxeter matrix are as following:

$C_{A}=\left(\begin{array}{llllll}1 & 1 & 1 & 1 & 1 & 2 \\ 0 & 1 & 0 & 0 & 0 & 1 \\ 0 & 0 & 1 & 0 & 0 & 1 \\ 0 & 0 & 0 & 1 & 0 & 1 \\ 0 & 0 & 0 & 0 & 1 & 1 \\ 0 & 0 & 0 & 0 & 0 & 1\end{array}\right), \Phi_{A}=\left(\begin{array}{cccccc}-1 & -1 & -1 & -1 & -1 & -2 \\ 0 & 1 & 0 & 0 & 0 & -1 \\ 0 & 0 & 1 & 0 & 0 & -1 \\ 0 & 0 & 0 & 1 & 0 & -1 \\ 0 & 0 & 0 & 0 & 1 & -1 \\ 0 & 0 & 0 & 0 & 0 & 1\end{array}\right)$

For each object $\quad X \in \underline{\bmod } \widehat{A}, \quad$ denote

$$
\underline{\operatorname{dim}}^{A} X=\left(x_{1}, x_{2}, \cdots, x_{6}\right) \text {, }
$$

Then $\chi_{A}\left(\underline{\operatorname{dim}}^{A} X\right)=\sum_{i=2}^{5}\left(x_{i}-\frac{x_{1}+x_{6}}{2}\right)^{2}$.

(1) Description of the minimal imaginary root $\delta$.

Case 1. If $r_{0}+r_{\infty} \equiv 1(\bmod 2)$

$\delta_{1}=\left(2 r_{0}, r_{0}+r_{\infty}, r_{0}+r_{\infty}, r_{0}+r_{\infty}, r_{0}+r_{\infty}, 2 r_{\infty}\right)$.

Case 2. If $r_{0}+r_{\infty} \equiv 0(\bmod 2)$

$\delta_{2}=\left(r_{0}, \frac{r_{0}+r_{\infty}}{2}, \frac{r_{0}+r_{\infty}}{2}, \frac{r_{0}+r_{\infty}}{2}, \frac{r_{0}+r_{\infty}}{2}, r_{\infty}\right)$.

(2) Description of $\underline{\operatorname{dim}}^{A} X$ where $X$ is an object in the bottom of a tube of rank 2 .

If $\delta=\delta_{1}$, that is $r_{0}+r_{\infty} \equiv 1(\bmod 2),(*)$ in Theorem 1.6 should be as follows:

$$
\left\{\begin{array}{c}
\sum_{i=1}^{6} x_{i}^{2}-\sum_{i=2}^{5} x_{1} x_{i}-\sum_{i=2}^{5} x_{6} x_{i}+2 x_{1} x_{6}=1 \\
\sum_{i=2}^{5} x_{i}-2 x_{6}=2 r_{0} \\
\sum_{i=2}^{5} x_{i}-x_{1}-x_{6}=r_{0}+r_{\infty} \\
\sum_{i=2}^{5} x_{i}-2 x_{1}=2 r_{\infty}
\end{array}\right.
$$


$\underline{\mathrm{d} \mathrm{m}}^{A} X=\left(\dot{\eta_{0}}, \frac{r_{0}+r_{\infty}-1}{2}, \frac{r_{0}+r_{\infty}-1}{2}\right.$,

$$
\left.\frac{r_{0}+r_{\infty}+1}{2}, \frac{r_{0}+r_{\infty}+1}{2}, r_{\infty}\right)
$$

$\underline{\mathrm{d} \mathrm{m}}^{A} \hat{\tau} X=\left(1 r_{0}, \frac{r_{0}+r_{\infty}+1}{2}, \frac{r_{0}+r_{\infty}+1}{2}\right.$,

$$
\left.\frac{r_{0}+r_{\infty}-1}{2}, \frac{r_{0}+r_{\infty}-1}{2}, r_{\infty}\right)
$$

(ii)

$$
\begin{aligned}
\underline{\operatorname{dim}}^{A} X= & \left(r_{0}, \frac{r_{0}+r_{\infty}-1}{2}, \frac{r_{0}+r_{\infty}+1}{2},\right. \\
& \left.\frac{r_{0}+r_{\infty}-1}{2}, \frac{r_{0}+r_{\infty}+1}{2}, r_{\infty}\right)
\end{aligned}
$$

$\underline{\operatorname{dim}}^{A} \hat{\tau} X=\left(r_{0}, \frac{r_{0}+r_{\infty}+1}{2}, \frac{r_{0}+r_{\infty}-1}{2}\right.$,

$$
\left.\frac{r_{0}+r_{\infty}+1}{2}, \frac{r_{0}+r_{\infty}-1}{2}, r_{\infty}\right)
$$

(iii)

$\underline{\operatorname{dim}}^{A} X=\left(r_{0}, \frac{r_{0}+r_{\infty}-1}{2}, \frac{r_{0}+r_{\infty}+1}{2}\right.$,

$$
\left.\frac{r_{0}+r_{\infty}+1}{2}, \frac{r_{0}+r_{\infty}-1}{2}, r_{\infty}\right)
$$

$\underline{\operatorname{dim}}^{A} \hat{\tau} X=\left(r_{0}, \frac{r_{0}+r_{\infty}+1}{2}, \frac{r_{0}+r_{\infty}-1}{2}\right.$,

$$
\left.\frac{r_{0}+r_{\infty}-1}{2}, \frac{r_{0}+r_{\infty}+1}{2}, r_{\infty}\right)
$$

(iv)

$$
\begin{aligned}
\underline{\mathrm{d} \mathrm{m}}^{A} X= & \left(\dot{r}_{\mathrm{b}}+1, \frac{r_{0}+r_{\infty}+1}{2}, \frac{r_{0}+r_{\infty}+1}{2},\right. \\
& \left.\frac{r_{0}+r_{\infty}+1}{2}, \frac{r_{0}+r_{\infty}+1}{2}, r_{\infty}+1\right) \\
\underline{\mathrm{d} \mathrm{m}}^{A} \hat{\tau} X= & \left(1 r_{0}-1, \frac{r_{0}+r_{\infty}-1}{2}, \frac{r_{0}+r_{\infty}-1}{2},\right. \\
& \left.\frac{r_{0}+r_{\infty}-1}{2}, \frac{r_{0}+r_{\infty}-1}{2}, r_{\infty}-1\right)
\end{aligned}
$$

If $\delta=\delta_{2},(*)$ in Theorem 1.6 should be as follows:

$$
\begin{aligned}
& \left(\sum_{i=1}^{6} x_{i}^{2}-\sum_{i=2}^{5} x_{1} x_{i}-\sum_{i=2}^{5} x_{6} x_{i}+2 x_{1} x_{6}=1\right. \\
& \sum_{i=2}^{5} x_{i}-2 x_{6}=r_{0} \\
& \sum_{i=2}^{5} x_{i}-x_{1}-x_{6}=\frac{r_{0}+r_{\infty}}{2} \\
& \sum_{i=2}^{5} x_{i}-2 x_{1}=r_{\infty} \\
& \text { Then, } \sum_{i=2}^{5}\left(x_{i}-\frac{r_{0}+r_{\infty}{ }^{2}}{4}\right)^{2}=1 \text {. }
\end{aligned}
$$

case 2. When $r_{0}+r_{l} \equiv 2(\bmod 4)$, we have four different tubes of rank 2 .

(i)

$$
\begin{aligned}
\underline{\operatorname{dim}}^{A} X= & \left(\frac{r_{0}-1}{2}, \frac{r_{0}+r_{\infty}+2}{4}, \frac{r_{0}+r_{\infty}-2}{4},\right. \\
& \left.\frac{r_{0}+r_{\infty}-2}{4}, \frac{r_{0}+r_{\infty}-2}{4}, \frac{r_{\infty}-1}{2}\right)
\end{aligned}
$$

$\underline{\operatorname{dim}}^{A} \hat{\tau} X=\left(\frac{r_{0}+1}{2}, \frac{r_{0}+r_{\infty}-2}{4}, \frac{r_{0}+r_{\infty}+2}{4}\right.$,

$$
\left.\frac{r_{0}+r_{\infty}+2}{4}, \frac{r_{0}+r_{\infty}+2}{4}, \frac{r_{\infty}+1}{2}\right)
$$

(ii)

$$
\begin{aligned}
\underline{\operatorname{dim}}^{A} X= & \left(\frac{r_{0}-1}{2}, \frac{r_{0}+r_{\infty}-2}{4}, \frac{r_{0}+r_{\infty}+2}{4},\right. \\
& \left.\frac{r_{0}+r_{\infty}-2}{4}, \frac{r_{0}+r_{\infty}-2}{4}, \frac{r_{\infty}-1}{2}\right)
\end{aligned}
$$

$\underline{\operatorname{dim}}^{A} \hat{\tau} X=\left(\frac{r_{0}+1}{2}, \frac{r_{0}+r_{\infty}+2}{4}, \frac{r_{0}+r_{\infty}-2}{4}\right.$,

$$
\left.\frac{r_{0}+r_{\infty}+2}{4}, \frac{r_{0}+r_{\infty}+2}{4}, \frac{r_{\infty}+1}{2}\right)
$$

(iii)

$$
\begin{aligned}
\underline{\mathrm{d} \mathrm{m}}^{A} X= & \left(\frac{r_{0}-1}{2}, \frac{r_{0}+r_{\infty}-2}{4}, \frac{r_{0}+r_{\infty}-2}{4},\right. \\
& \left.\frac{r_{0}+r_{\infty}+2}{4}, \frac{r_{0}+r_{\infty}-2}{4}, \frac{r_{\infty}-1}{2}\right)
\end{aligned}
$$

$$
\begin{aligned}
\mathrm{d} \mathrm{m}^{A} \hat{\tau} X= & \left(\frac{r_{0}+1}{2}, \frac{r_{0}+r_{\infty}+2}{4}, \frac{r_{0}+r_{\infty}+2}{4},\right. \\
& \left.\frac{r_{0}+r_{\infty}-2}{4}, \frac{r_{0}+r_{\infty}+2}{4}, \frac{r_{\infty}+1}{2}\right)
\end{aligned}
$$

(iv) 


$$
\begin{aligned}
\underline{\mathrm{d} \mathrm{m}}^{A} X= & \left(\frac{r_{q}-1}{2}, \frac{r_{0}+r_{\infty}-2}{4}, \frac{r_{0}+r_{\infty}-2}{4},\right. \\
& \left.\frac{r_{0}+r_{\infty}-2}{4}, \frac{r_{0}+r_{\infty}+2}{4}, \frac{r_{\infty}-1}{2}\right) \\
\frac{\mathrm{d} \mathrm{m}}{} \hat{\tau} X= & \left(\frac{r_{0}+1}{2}, \frac{r_{0}+r_{\infty}+2}{4}, \frac{r_{0}+r_{\infty}+2}{4},\right. \\
& \left.\frac{r_{0}+r_{\infty}+2}{4}, \frac{r_{0}+r_{\infty}-2}{4}, \frac{r_{\infty}+1}{2}\right)
\end{aligned}
$$

case 3. When $r_{0}+r_{l} \equiv 0(\bmod 4)$, we have four different tubes of rank 2 .

(i)

$$
\begin{aligned}
& \underline{\mathrm{d} \mathrm{m}}^{A} X \dot{\mp}\left(\frac{r_{0}+1}{2}, \frac{r_{0}+r_{\infty}}{4}, \frac{r_{0}+r_{\infty}}{4}\right. \text {, } \\
& \left.\frac{r_{0}+r_{\infty}}{4}, \frac{r_{0}+r_{\infty}+4}{4}, \frac{r_{\infty}+1}{2}\right) \\
& \underline{\mathrm{d} \mathrm{m}}^{A} \hat{\tau} X \mathrm{i}=\left(\frac{r_{0}-1}{2}, \frac{r_{0}+r_{\infty}}{4}, \frac{r_{0}+r_{\infty}}{4},\right. \\
& \left.\frac{r_{0}+r_{\infty}}{4}, \frac{r_{0}+r_{\infty}-4}{4}, \frac{r_{\infty}-1}{2}\right)
\end{aligned}
$$

(ii)

$$
\begin{aligned}
& \underline{\mathrm{d} \mathrm{m}}^{A} X \dot{\mathrm{m}}\left(\frac{r_{0}+1}{2}, \frac{r_{0}+r_{\infty}}{4}, \frac{r_{0}+r_{\infty}}{4},\right. \\
&\left.\frac{r_{0}+r_{\infty}+4}{4}, \frac{r_{0}+r_{\infty}}{4}, \frac{r_{\infty}+1}{2}\right) \\
& \frac{\mathrm{d} \mathrm{m}}{} \hat{\tau} X \mathrm{i}=\left(\frac{r_{0}-1}{2}, \frac{r_{0}+r_{\infty}}{4}, \frac{r_{0}+r_{\infty}}{4},\right. \\
&\left.\frac{r_{0}+r_{\infty}-4}{4}, \frac{r_{0}+r_{\infty}}{4}, \frac{r_{\infty}-1}{2}\right)
\end{aligned}
$$

(iii)

$$
\begin{aligned}
\underline{\operatorname{dim}}^{A} X= & \left(\frac{r_{0}+1}{2}, \frac{r_{0}+r_{\infty}}{4}, \frac{r_{0}+r_{\infty}+4}{4},\right. \\
& \left.\frac{r_{0}+r_{\infty}}{4}, \frac{r_{0}+r_{\infty}}{4}, \frac{r_{\infty}+1}{2}\right) \\
\underline{\operatorname{dim}}^{A} \hat{\tau} X= & \left(\frac{r_{0}-1}{2}, \frac{r_{0}+r_{\infty}}{4}, \frac{r_{0}+r_{\infty}-4}{4},\right. \\
& \left.\frac{r_{0}+r_{\infty}}{4}, \frac{r_{0}+r_{\infty}}{4}, \frac{r_{\infty}-1}{2}\right)
\end{aligned}
$$

(iv)

$$
\begin{aligned}
\underline{\operatorname{dim}}^{A} X= & \left(\frac{r_{0}+1}{2}, \frac{r_{0}+r_{\infty}+4}{4}, \frac{r_{0}+r_{\infty}}{4},\right. \\
& \left.\frac{r_{0}+r_{\infty}}{4}, \frac{r_{0}+r_{\infty}}{4}, \frac{r_{\infty}+1}{2}\right)
\end{aligned}
$$

$$
\begin{aligned}
\underline{\operatorname{dim}}^{A} \hat{\tau} X= & \left(\frac{r_{0}-1}{2}, \frac{r_{0}+r_{\infty}-4}{4}, \frac{r_{0}+r_{\infty}}{4},\right. \\
& \left.\frac{r_{0}+r_{\infty}}{4}, \frac{r_{0}+r_{\infty}}{4}, \frac{r_{\infty}-1}{2}\right)
\end{aligned}
$$

\section{Acknowledgment}

The authors would like to thank the referee for his or her valuable suggestions and comments. The first-named author thanks NSF of China (Grant No. 11126300) and of Shandong Province (Grant No. ZR2011AL015) for support.

\section{REFERENCES}

[1] C.M.Ringel, Tame algebras and integral quadratic forms, Lecture Notes in Math. 1099. Springer Verlag, 1984.

[2] D.Happel, Triangulated categories in the representation theory of finite dimensional algebras, Lecture Notes series 119. Cambridge Univ. Press, 1988.

[3] D.Happel, On the derived category of a finitedimensional algebra. Comment. Math. Helv. 62(1987), 339_389.

[4] D.Happel, C.M.Ringel, The derived category of a tubular

algebra. LNM1273, Berlin-Heidelbelrg-NewYork: Springer-Verlag, 1986:156_180.

[5] W.Crawley-Boevey, Lectures on Representations of Quivers. Preprint. 